\title{
Parasuicidio en púberes y adolescentes escolarizados
}

\section{Parasuicide in puberty and school adolescents}

\author{
QUINTERO Amparo ${ }^{1}$ \\ SÁNCHEZ Liliana ${ }^{2}$ \\ AGREDO Jobana ${ }^{3}$ \\ OVIEDO Liliana ${ }^{4}$
}

\begin{abstract}
Resumen
El presente estudio, con enfoque cualitativo de tipo exploratorio-descriptivo, tiene como objetivo identificar y describir los pensamientos, emociones y conductas de 21 adolescentes, de ambos sexos con edades entre 11 y 17 años, quienes habían presentado al menos una conducta de autoagresión. Se aplicaron de forma individual el Instrumento de Riesgo Suicida Adolescente IRSA, la Escala de Intencionalidad Suicida de Beck, y el Cuestionario sociodemográfico. En el análisis descriptivo se calcularon frecuencias, porcentajes, medias, medianas y rangos. Los resultados mostraron que el grupo de participantes tendía a legitimar las diversas autolesiones como parte de su expresión de individualidad, y que el tipo de interacción familiar y las condiciones académicas pueden funcionar como factores de riesgo o de protección.

Palabras clave: parasuicidio, adolescentes, familia, ambiente escolar.
\end{abstract}

\begin{abstract}
This characterization study aimed to identify and describe the thoughts, emotions and behaviors of 21 adolescents, of both sexes between the ages of 11 and 17, who had issued at least one self-aggression behavior. The IRSA Teen Suicidal Risk Instrument, Beck's Suicide Intentionality Scale, and the Sociodemographic Questionnaire. The descriptive analysis calculated frequencies, percentages, averages, medians, and ranges. The results showed that the group of participants tended to legitimize the various self-harms as part of their expression of individuality, and that the type of family interaction and academic conditions may apparently function as risk or protection factors depending on the actual level of family harmony and academic performance.
\end{abstract}

Keywords: parasuicide, adolescents, family, school environment.

\section{Introducción}

Datos mundiales señalan a el suicidio y el parasuicidio como dos de los problemas más importantes que aquejan a la población actual, en especial en países de América Latina incluido Colombia (Organización Mundial de la Salud, OMS, 2019). El informe del Ministerio de Salud en Colombia reconoce que el suicidio ha sido un problema de salud pública, razón por la que lo incluye como uno de los principales temas de vigilancia prioritaria del Plan Decenal de Salud Pública 2012-2021 (Ministerio de Salud y Protección Social, 2012).

\footnotetext{
${ }^{1}$ Docente Ocasional Hora Catedra, ECSAH. UNAD. Amparo.quintero@unad.edu.co

2 Docente Ocasional Tiempo Completo, ECSAH. UNAD. Liliana.sanchez@unad.edu.co

${ }^{3}$ Docente Ocasional Tiempo Completo, ECSAH. UNAD. Jobana.agredo@unad.edu.co

${ }^{4}$ Docente Ocasional Tiempo Completo, ECSAH. UNAD. Liliana.oviedo@unad.edu.co
} 
En el caso específico de los adolescentes se presentan conductas autolesivas que en ocasiones alcanzan tal magnitud que se clasifican como acciones parasuicidas, en las que la persona se autoagrede con el fin de dar aviso a otros acerca de la propia necesidad de ayuda para solucionar los conflictos que vive en sus entornos; realidades que a su vez denotan la importancia de comprender su transformación generacional debido a las modificación de las condiciones psicológicas y los contextos familiares, escolares y mediáticos (Soto-Salcedo, Villaroel Grüner, \& Véliz Burgos, 2020), ya que sin duda las nuevas formas de vivir y afrontar la realidad afectan la salud mental de púberes y adolescentes (Red de Salud de Cuba [INFOMED], 2018; Rodríguez Molinero, 2017; Sanderson, 2020).

El fenómeno de la autolesión en los adolescentes ha generado una alta aproximación analítica debido a que el parasuicidio es un acontecimiento que actualmente afecta a la población adolescente en un buen número de países a nivel mundial, más allá del nivel socioeconómico y cultural, ya que es una experiencia individual determinada por causas, entornos y estrategias diferentes en el que se infringen las autoagresiones (Gaviria y Torres, 2019). Es así como la OMS, en 2018, lidera la Iniciativa Mundial para la Prevención del Suicidio (SUPRE), la cual incluye diversos lineamientos técnicos dirigidos a diferentes actores, entre los que se cuentan médicos generales, profesionales de los medios de comunicación, docentes y otro personal institucional, trabajadores de la atención primaria en salud, policías, bomberos y otros socorristas de primera línea; ofrece orientaciones para la puesta en marcha de acciones de intervención realizadas en diferentes entornos como las instituciones educativas, las cárceles y a otras personas afectadas por el fenómeno, que son consideradas supervivientes.

Adicionalmente, es importante señalar que cada año se suicida 1 millón de personas en todo el mundo, y que la tasa de mortalidad global es de 16 por 100.000, o una muerte de este tipo cada 40 segundos; y que además que las muertes por suicidio en el mundo han aumentado en un 60\% en los últimos 45 años, y está entre las primeras causas de muerte entre los 15 y 44 años de edad; cifras que la OMS (2014) atendió y en respuesta creó el Programa de Acción para Superar las Brechas en Salud Mental, mhGAP, en el que incluyó entre las acciones en pro de salud mental priorizadas un el módulo de entrenamiento, dirigido a personal de salud no especializado en los servicios de baja complejidad, denominado "Autolesión/Suicidio" como posibilidad en la mejora de la detección y manejo oportuno de casos de riesgo.

Específicamente en Colombia, de 2005 a 2014, el sistema médico legal registró 18.336 casos de suicidio, cifras que indican que al menos 1.833 personas se suicidaron cada año en el país (Domínguez, 2015); cifras que para 2019 muestran que esta problemática no ha tenido un panorama esperanzador en especial para la población adolescente (Instituto Nacional de Medicina Legal y Forenses, 2019).

En respuesta a esta realidad surge la necesidad de indagar las características y las causas del parasuicidio en los estudiantes púberes y adolescentes de instituciones educativas públicas en la ciudad de Ibagué, desde una mirada cognitiva, emocional y conductual en contextos familiar, escolar y personal; lo cual se justifica con ésta población porque el suicidio va en aumento de forma alarmante: en 2016 fue la segunda causa principal de defunción en el grupo etario de 15 a 29 años en todo el mundo y más del 79\% de los suicidios tuvieron lugar en países de ingresos bajos y medianos (OMS, 2018).

Por otro lado, en el informe regional respecto al suicidio en las Américas, divulgado por la Organización Panamericana de la Salud y la Organización Mundial de la Salud (OPS/OMS, 2014; OMS, 2018), Colombia registra una tasa de 4.54 suicidios por cada 100.000 habitantes, lo que la ubica en el lote intermedio a nivel regional. Esta preocupante situación se da principalmente en ciudades capitales, como es el caso de lbagué tal como lo ratifica el Departamento Administrativo Nacional de Estadística (DANE, 2017). Es importante señalar que por cada suicidio consumado se han dado de 8 a 10 intentos, y que, por cada intento, 8 lo pensaron, planearon y estuvieron a punto de hacerlo (Beaven et al., 2018); y que al menos el $40 \%$ de quienes han intentado suicidarse lo han hecho en varias ocasiones (González et al., 2016); razones por las que se hace urgente e importante 
estudiar el proceso que antecede a los suicidios para así conocer y atender este problema de alta presencia en la adolescencia.

\subsection{Rango etáreo de alto riesgo}

Durante la adolescencia cada persona adquiere su identidad y la posición individual intergeneracional, proceso en el que la familia juega un papel verdaderamente importante, y proporciona las principales funciones de socialización entre pares, condiciones que constituyen el ámbito básico en el que se da el desarrollo y facilita el bienestar en uno de los más importantes momentos de la vida (Monge, 2013), en especial porque se enfrentan diversas dificultades debido a que durante la adolescencia se presenta alta impulsividad y reactividad emocional, rasgos de personalidad que incrementan el riesgo de emitir conductas con fines de autolesión que no siempre tienen propósitos suicidas (De la Fuente, 2018; Acevedo, Lizcano y Serrano, 2020).

Impulsividad que involucra la urgencia de acción en respuesta a condiciones personales como baja autoestima, alta crítica a sí mismo, sentimientos negativos de fracaso, inferioridad, agresividad, dificultades para la expresión e identificación de sus emociones (alexitimia) y sentimientos negativos en especial hacia sí mismos, entre otras condiciones características que presenta un número importante de personas que se hacen daño y presentan trastornos psiquiátricos entre los que se cuentan el consumo de alcohol y drogas (Tomko, Prisciandaro, Falls, \& Magid, 2016; Acevedo et al., 2020).

Las ideaciones de muerte y /o daño de los adolescentes muestran que existen problemas de salud mental importantes en este grupo poblacional, expresados en conductas disruptivas como (hiperactividad en clase, atención dispersa y rebeldía ante los padres) y relacionados con la presencia de ideación de muerte de personas cercanas, ideas de muerte de mascotas, ideas acerca de su propia muerte y acciones respecto como hacerse daño, además de ideaciones suicidas activas; condiciones con mayor reporte cuando se registra historia previa de conducta suicida y daño a sí mismo o a otros en los últimos 12 meses (Colón, 2019). Además, es preciso anotar que la mayor parte de adolescentes que emiten conductas de daño a sí mismos son hombres. Situación que ocurre en los mismos términos en Ibagué tal como se aprecia, aunque se evidencian disminuciones temporales entre 2013 y 2015, año en el que se prácticamente se duplicó (Figura 1).

Figura 1

TASA DE SUICIDIOS EN ADOLESCENTESDE 12 A 17 AÑOS

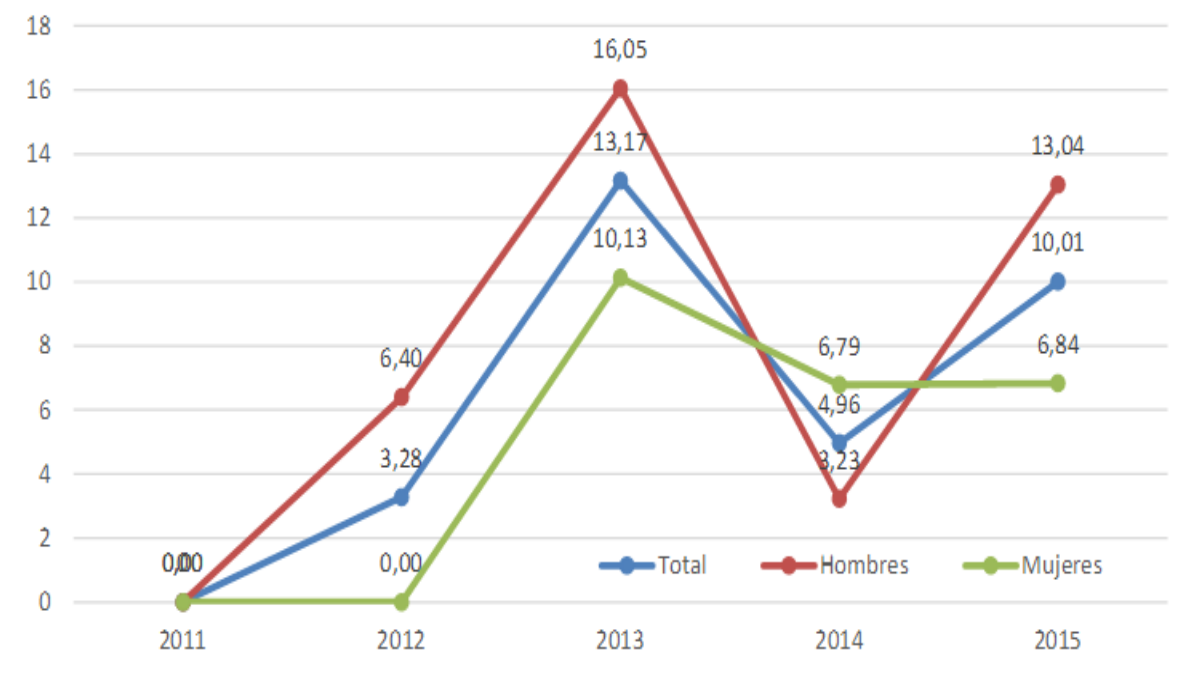

Fuente: Secretaria de Salud de Ibagué, 2016 
El fenómeno de autolesionarse, es uno de los problemas sociales y de salud mental, más visible y polémico a nivel mundial; por lo tanto, es necesario comprender este fenómeno en la población adolescente y las formas en que se origina y se mantiene en algunos sectores; por supuesto sin desconocer las particularidades de los adolescentes como personas en construcción, que interpretan el mundo y lo reconstruyen a diario.

Es importante anotar que, el intento de causar la propia muerte es una etapa del proceso suicida, que en el caso especial de los adolescentes tiene un alto impacto en la población en general, ocurre de manera cada vez más frecuente debido a que viven un momento considerado de extrema vulnerabilidad debido a la falta de seguridad y pobre control emocional, incluso en población escolarizada (Suárez Colorado y Campo-Arias, 2019; Londoño Muriel y Cañón Buitrago, 2020). Sin lugar a dudas es necesario aportar a la comprensión de conductas autolesivas en población en el inicio y cierre de este momento especial en el proceso de desarrollo, por ello es preciso caracterizar pensamientos, emociones y conductas de adolescentes que emiten conductas de autolesión.

\section{Metodología}

\subsection{Población}

En el presente estudio, con enfoque cualitativo de tipo exploratorio-descriptivo, participaron 21 estudiantes con antecedentes de conductas de autolesión, con edades entre 12 y 17 años, pertenecientes a 2 instituciones de educación media de la ciudad de lbagué (Colombia), quienes fueron identificados por los respectivos psicólogos de dichas instituciones (Tabla 1).

Tabla 1

\begin{tabular}{|c|c|c|c|c|}
\hline \multicolumn{5}{|c|}{ Caracterización de la población } \\
\hline Participante & Nombre & Edad en años & Sexo & Grado \\
\hline 1 & E. U. & 12 & Mujer & 60 \\
\hline 2 & M. G. & 12 & Mujer & 60 \\
\hline 3 & V. A. & 11 & Mujer & 60 \\
\hline 4 & L. C. & 13 & Mujer & 60 \\
\hline 5 & K. D. & 12 & Hombre & 7음 \\
\hline 6 & N.S. & 13 & Mujer & 7으 \\
\hline 7 & A. T. & 13 & Mujer & 7으 \\
\hline 8 & D. Z. & 14 & Hombre & 70 \\
\hline 9 & B. G. & 13 & Hombre & 7음 \\
\hline 10 & A. $M$. & 15 & Mujer & 7음 \\
\hline 11 & Y. C. & 14 & Mujer & 7음 \\
\hline 12 & A. $R$. & 14 & Mujer & 70 \\
\hline 13 & Y. M. & 14 & Mujer & 70 \\
\hline 14 & A. A. & 14 Años & Mujer & 70 \\
\hline 15 & G. U. & 12 años & Hombre & 80 \\
\hline 16 & B. $\mathrm{H}$. & 13 años & Mujer & 80 \\
\hline 17 & J.R. & 17 años & Hombre & 80 \\
\hline 18 & A.P. & 15 años & Mujer & 80 \\
\hline 19 & B. V. & 14 años & Hombre & 9o \\
\hline 20 & Y. T. & 17 años & Hombre & 9o \\
\hline 21 & K. R. & 15 años & Mujer & $10 \circ$ \\
\hline
\end{tabular}




\subsection{Enfoque de Investigación}

Para alcanzar los objetivos propuestos, se consideraron los parámetros metodológicos desde un enfoque mixto ya que se usaron instrumentos validados para recolectar la información principal con garantía de calidad psicométrica; para luego, completar el análisis, bajo una mirada cualitativa basada en la información recogida en una entrevista individual a profundidad, la cual enfatiza el estudio de los procesos y significados, interesándose por los fenómenos y la comprensión de las experiencias humanas (Vasilachis, 2006).

\subsection{Tipo de Investigación}

La investigación, exploratoria - descriptiva, se enfocó sobre un tema u objeto desconocido o poco estudiado, por lo que sus resultados constituyen una visión aproximada de dicho objeto. Esta es un tipo de investigación que comprende al sujeto de una manera individualizada, es decir que no lo adapta a categorías preconcebidas (Sandoval, 2010).

\subsection{Técnicas e Instrumentos}

En la investigación se aplicaron los siguientes instrumentos de medición:

El Instrumento de Riesgo Suicida Adolescente IRISA ( Anexo 1), elaborada por Hernández y Lucio (2006) y validada para población colombiana por Alarcón-Velásquez et al. (2019), cuyo objetivo es medir y registrar los factores de riesgo suicida en adolescentes, para ello evalúa 7 ámbitos diferentes: Factores de riesgo no modificables, factores modificables, depresión /ansiedad, ideación suicida, factores precipitantes, creencias y factores protectores. Este instrumento tiene alto nivel de consistencia global $(\alpha=.962)$ y por escalas que varía entre una de .798 a .966 .

La Escala de Intencionalidad Suicida de Beck (1979) (Suicide Intent Scale, SIS) traducida al español y validada por García-Nieto et al. (2003), tiene como objetivo evaluar las características de la tentativa suicida: circunstancias en las que se lleva a cabo, actitud hacia la vida y la muerte, pensamientos y conductas antes, durante y después de la tentativa de suicido, consumo de alcohol u otras sustancias relacionada con la tentativa. Consta de 3 partes diferenciadas: parte objetiva, parte subjetiva, y otros aspectos. Consta de 15 ítems calificados de 0 a 2, cuya suma de puntajes por encima de 16.3 indica de gravedad media y alta; y cuenta con altos índices de fiabilidad y validez (Anexo 2). Y el Cuestionario sociodemográfico y áreas de ajuste cognitiva, emocional y conductual, el cual fue diseñado para el presente proyecto de investigación aplicado en entrevista individual ( Anexo 3).

\section{Resultados y discusión}

A continuación, se presentan los resultados descriptivos obtenidos con cada uno de los instrumentos y sus respectivos factores de evaluación; también estadísticos básicos descriptivos que incluyeron frecuencias, porcentajes, medias y rangos, representaciones gráficas de las variables más relevantes y principales insumos para la realización de la investigación. Para la codificación de la información durante el proceso de análisis de los datos en algunas tablas, se escriben las iniciales de los nombres de los participantes.

\subsection{Estructura familiar}

Familias pertenecientes a los estratos 1 y 2, el $62 \%$ posee ocupación informal y sólo un $24 \%$ es formal. En la figura 1 , se observa que el promedio más elevado de participantes que experimentan la conducta parasuicida se encuentran dentro de la estructura familiar de familia extensa, representando el $43 \%$ de la población total, familia nuclear con un $24 \%$. Seguido por el rango de estructura familiar mixta con un $14 \%$. Por último, con un $10 \%$ los participantes dentro del rango de familia monoparental. 
Figura 2

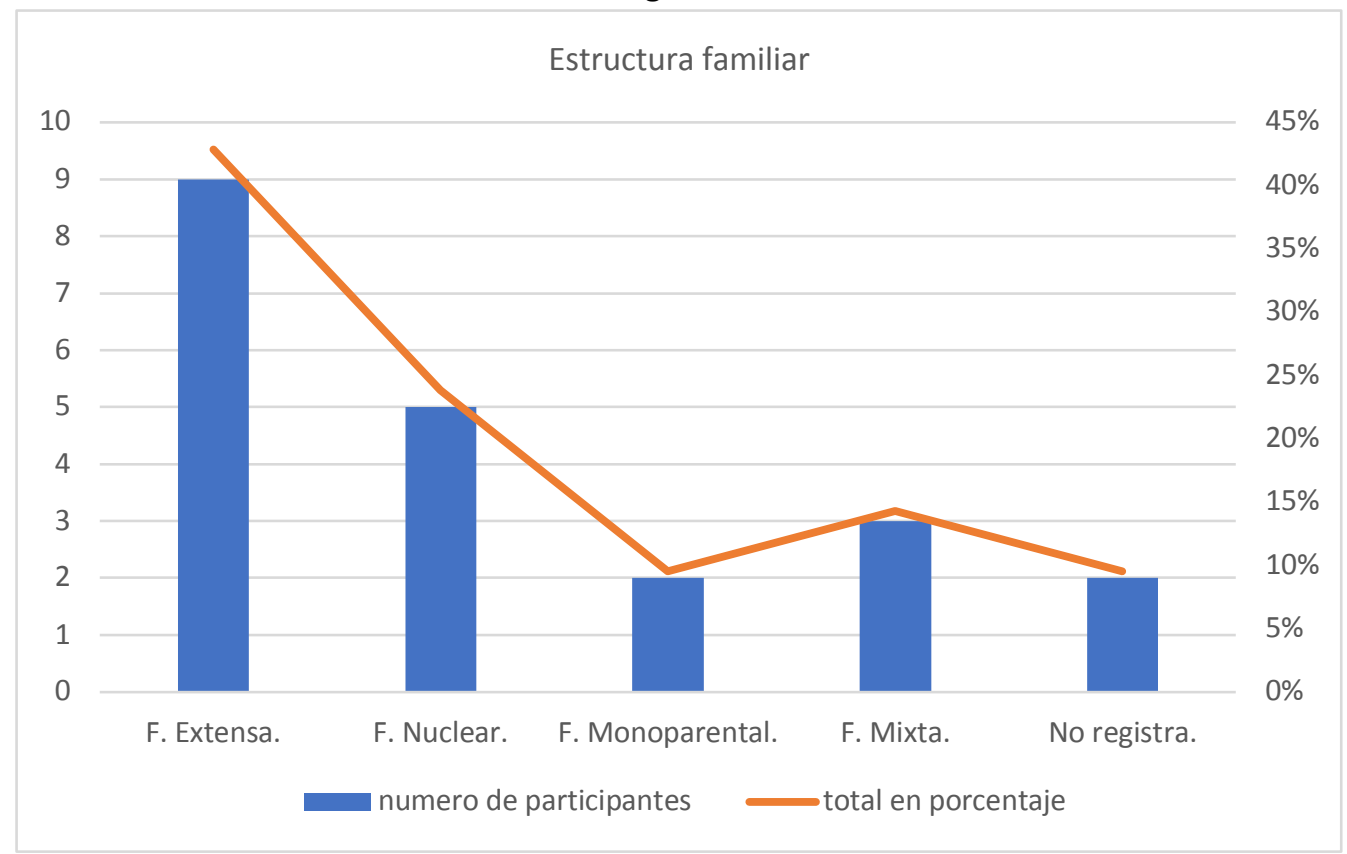

\subsection{Datos personales}

\subsubsection{Género}

La muestra seleccionada de participantes con experiencia parasuicida estaba principalmente conformada por 14 mujeres (67\%) y 7 hombres (33\%), aunque es preciso anotar que todos los casos fueron considerados con el mismo nivel de importancia. En general, es posible afirmar que existe una mayor frecuencia en mujeres del total de la muestra y una proporción de 2 a 1, en comparación de los hombres (Figura 3).

Figura 3

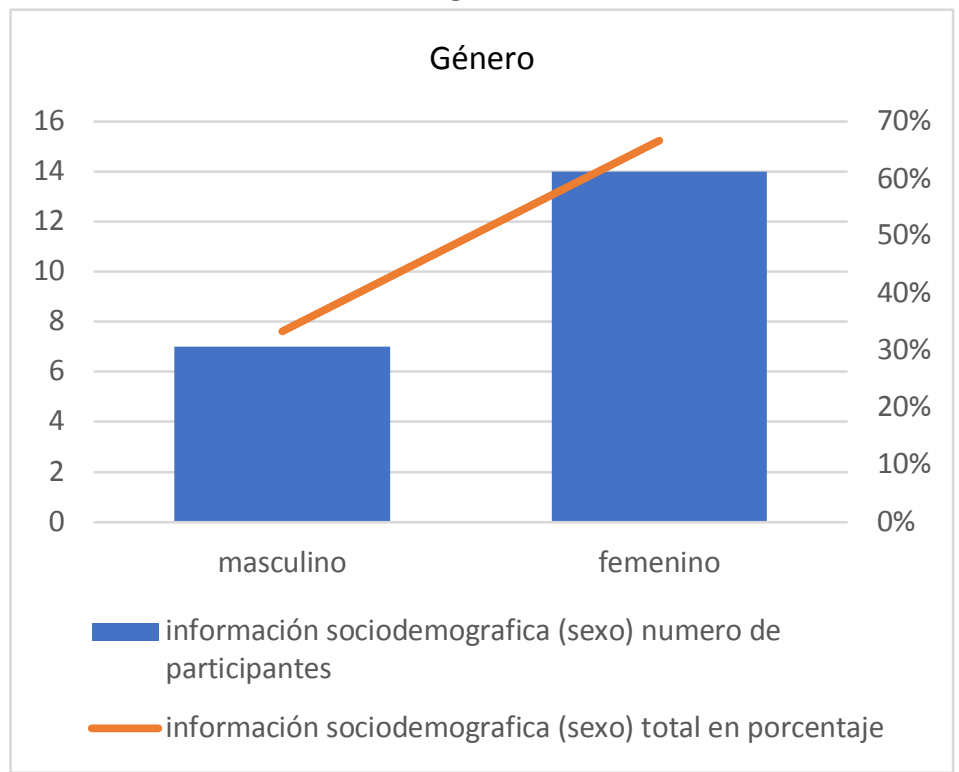

\subsubsection{Rango de edad}

En la Tabla 2. Los participantes que reportan haber tenido experiencia parasuicida a los 11 años es de (un 5\%); siendo el rango de mayor riesgo aumentando significativamente en las edades entre los 12 (un 19\%), 13 años 
(un $25 \%$ ), 14 años (un 29\%); disminuyendo de forma significativa en la edad en el tramo entre los 15 (un $14 \%$ )y 17 años de edad (un 10\%).

Tabla 2

Frecuencia de experiencia parasuicida y edad.

$\begin{array}{ccc}\begin{array}{c}\text { Edad en } \\ \text { años }\end{array} & \text { Frecuencia } & \text { Porcentaje } \\ 11 & 1 & 4 \% \\ 12 & 4 & 19 \% \\ 13 & 5 & 24 \% \\ 14 & 6 & 29 \% \\ 15 & 3 & 14 \% \\ 16 & 0 & 0 \% \\ 17 & 2 & 10 \% \\ \text { Total } & 21 & 100 \%\end{array}$

\subsection{Características de la conducta parasuicida (autolesión)}

A partir de los resultados de la entrevista semiestructurada, los resultados de la prueba de Beck y el test de IRSA se logró identificar la presencia de las siguientes conductas de autolesión: mayoritariamente de cutting (corte) $71.4 \%$, seguido por ingesta de sustancias tóxicas $23.8 \%$, insecticidas, medicamentos neurológicos y benceno e intento de ahorcamiento 4.7\%; estas acciones son sustentadas desde las siguientes dimensiones, buscando desde sus voces visibilizar el fenómeno del parasuicidio en los 21 sujetos así:

\subsubsection{Dimensión cognitiva}

De los 21 evaluados 10 participantes responden no saber información sobre conductas autolesivas, según sus verbalizaciones al respecto: "Nunca me ha preocupado buscar información", "Ninguna, solo lo hago y ya"; así mismo 9 participantes refieren que hay muchas formas para hacerlo: "Mi mamá me ha leído sobre el Cutting, que es dañino, que me estoy perjudicando, acabando con mi vida lentamente", "Esto se da por muchos problemas, unos se matan, otros se cortan, se cuelgan, todo lo que uno hace es por problemas" y aunque saben que no deben hacerlo, de todas maneras lo hacen: 2 refieren "Se mucho, y también que no debo atentar contra la vida aunque yo lo hago".

Ante el cuestionamiento sobre cómo estarán los seres queridos sin ellos, 12 piensan que sus familias estarían mal sin ellos: "... porque mi hermano el que tiene ahora 22 años se colgó y fue muy feo, estuvimos muy mal, aunque lo rescataron a tiempo", "No, porque me extrañarían mucho, porque soy la única mujer en la familia" y sería muy feo si yo no existiera"; mientras otros 5 participantes afirman que las familias estarían mejor sin ellos; los 4 restantes tienen dudas, no saben qué puede pasar con sus familias.

Frente a los pensamientos que anteceden al hecho de la conducta autolesiva, 10 refieren tener malos recuerdos: "Angustia por la presión de mi padrastro y con todos, porque él quería que todo se hiciera como él quería", "Pensaba que debería hacerlo porque me sentía mejor si no estaban conmigo", "Recordaba las peleas que tenía con mi mamá por las cosas que tocaba hacer en la casa y los problemas todos los días en la casa"; 8 evaluados, reportaron pensamientos de ira: "Ira y hacerlo para decepcionar a mi mamá, solo ofendía a mi mamá", "Lo hice 2 veces por rabia, no tenía con quien desquitarme", "Vi la imagen del Facebook y me arriesgue a hacerlo", "Pienso en suicidarme con rabia y morir"; y los 3 restantes asocian los pensamientos a celos: "Me dan celos de mi hermano porque a él le dan de todo y cuando yo pido algo no me lo dan", "Recordaba a mi papá porque no lo tengo a mi lado, se fue con otra". 
Respecto a los pensamientos durante el hecho, 11 manifiestan tener ira: "Pensamientos de enojo traía los hechos a mi memoria mientras me cortaba" , "Pienso en que es mejor no haber existido porque no había mucha comunicación en mi familia", "Me da rabia que no me quieran" ; 3, piensan en la muerte: "Me quería morir", "Pensé que me podría morir, que me había cortado una vena no me dolió pero no pasó eso"; 4, no identifican ningún pensamiento: "Nada, sólo pensaba en tomarlas", "Sólo pienso en cortarme las venas", "Sólo pensaba en el dolor"; y los otros 3 tres, lo asocian a pensamientos de libertad: "Pienso en cosas que posiblemente no haré, me siento libre durante ese momento", "Pienso que voy a descansar o que puedo hacer lo que yo deseo".

Frente a los pensamientos después de la autolesión, 7 participantes, se arrepienten de haberlo hecho: "Pensé en que no debía haberlo hecho porque me sentía muy mal", "Me arrepiento de haberlo hecho porque hay posibilidad de arreglar las cosas", " $Y$ después me arrepiento y ya"; 5 reportan tranquilidad después del hecho "Descanso"; "Me encierro y quedo tranquila"; 6 valoran mal el daño que se hicieron: "Sé que me estoy haciendo daño", "Pensaba en el dolor que le ocasionaba a mi seres queridos", "Se me podía infectar, nos compartimos fotos con sangre".; mientras están en el procedimiento 2 piensan en la muerte : "Sólo pensaba en la muerte", "Me acuesto a dormir pensando que no me quieren y me puedo morir"; sólo un estudiante no reporta ningún pensamiento simultáneo al procedimiento: "Nada en especial, sigo conversando con mis dos amigos porque me emborracho con ellos".

En relación a la posibilidades de repetir la autolesión, 8 afirman reproducir la conducta: "Porque en la casa no cambian las cosas", "Porque estoy cansada con la vida", "Sí, por los problemas que nunca hacen falta, mi papá nunca me ha apoyado, mi mamá siempre me regaña y mis amigas no me quieren"; 10 dicen que no lo harían: "Porque me hago daño", "No porque pienso en mi futuro", "ya me veo bien, me veo siguiendo con el estudio"; y los 3 restantes no lo saben: "Depende como siga mi familia", "Qué tal que pierda el año". Es importante reportar otros comentarios que surgieron durante la entrevista: "Informa en la entrevista que ha planeado quitarse la vida mediante el lanzamiento desde un puente ya que se encuentra aburrida", "Expresa tener pensamientos constantes de quitarse la vida, y lo reafirma y lo ha planeado cuando se está cortando".

\subsubsection{Dimensión Emocional}

De los 21 participantes acerca de la percepción del afecto, 5 se sienten amados por mamá: "No me siento importante solo le importo a mi mamá"; 6 por la familia: "Sí, por mi familia con la que vivo y externas"; 3 por los amigos: "Si, por mis amigas, mi mamá más o menos", otros 3 por el novio/a y los 3 restantes reportaron desesperanza: "si me quisieran no me cortaría".

Acerca de los sentimientos antes de la conducta autolesiva, 8 sienten frustración o culpa: "Si me pasa algo más grave dejaría a mi papá sufriendo porque no le pude cumplir", "Frustración con rabia"; con sentimientos de soledad, 5 participantes: "Sola, vacía porque mi papá nunca ha vivido conmigo, nunca nos ha ayudado en lo económico", "Siento un vacío y a veces me pongo a llorar"; y con tristeza 8: "Tristeza, me deprimo", "Me siento muy mal, deprimido".

Entre los sentimientos reportados que presentan durante el hecho, en orden de frecuencia, estaban ira (5), tristeza (7), dolor (3), nervios (4), liberación (1) y nada (1).

A propósito de los sentimientos después del hecho, la tristeza se presenta en 9 participantes: "Entre tristeza y alegría.", "Triste hasta cuando me duermo"; 4, reportan sentimientos de culpa: "Culpa porque la embarré", "Me lamento" ; 5 sienten alivio: "Como que me relajo pero luego se me sube la tensión", "A veces pateo las almohadas y soy muy bipolar y se me vienen los recuerdos, me pongo triste y me distancio de todos, pero al final aliviada", "Siento descanso; y 3 refieren no sentir nada. 
Los contextos con los que relacionan los sentimientos de autolesión se presentan con mayor frecuencia alrededor de la familia, 14 casos: "Por la relación conflictiva con el ex-padrastro", "Todo fue por mi familia, porque mis papás peleaban mucho por la separación, peleaban porque mi mamá trabajaba de 12 a 12 y casi todas las noches llegaba llorando porque peleaba con mi papá", "Familia, sobre todo con mi papá, él tuvo un intento de suicidio, también he tenido a veces peleas con mi mamá y mi prima", "Me gusta cortarme, por mi abuela, ash ... es un fastidio"; 2, lo asocian con el colegio: "Por el Colegio, voy muy mal", "No quería volver a repetir el año"; 2, por los amigos: "Por mis amigos, son raros conmigo", "Yo lo hice por los amigos que se cortaban!"; y 3 refieren motivo por la pareja: "Por mi novio", "Es por mi novia por la ruptura, eso me dio muy duro".

\subsubsection{Dimensión conductual}

De los 21 púberes y adolescentes se encontró que 2 buscan información sobre la conducta autolesiva en redes sociales: "Una vez por Facebook, de unas cortadas, tengo el pantallazo en el celular", "en el Facebook encontré una imagen que decía f¿sabes por qué la gente se corta? Porque pueden soportar el dolor de sus cortadas, y no el de su corazónł, "yo lo hice no por el dolor del corazón sino por motivo de rabia"; 2 buscan información en internet: "Gogle, fotos", "Buscando una tarea, vi fotos"; 3 por amigos, "No he buscado información, pero mis amigos me han contado", "Nunca la he buscado, me enteré por amigas que lo hacían"; y la de mayor frecuencia, 14 refieren ninguna forma específica de buscar información al respecto: "No. Ninguna.", "No, nunca he buscado información".

Con referencia al comportamientos antes de la conducta autolesiva, 6 haberlo planeado: "Había planeado 2 semanas antes, había apartado el veneno (Neguvón); lo guarde en mi habitación", "Lo he hecho 2 veces uno en la casa, me encierro en el bañó y el otro fue en el colegio quedándome en el salón con un bisturí; 6 se aíslan: "Me acuesto en la cama a pensar", "Compro la cuchilla en la tiendo un día antes y sigo con mi comportamiento normal, pero me encierro para pensar mejor"; 7 buscan amigos: "Estaba con mis amigos porque ellos me dijeron que para poderlo hacer, tenía que tomarme esas pastillas y me las tomé en los baños del colegio", "Me siento a esperar a que lleguen mis amigos con el trago porque yo soy menor de edad y ellos si pueden comprar el trago"; 2 escuchan música con contenidos depresivos: "Estaba sola en la casa, ponía música de rap o música depresiva y buscaba la minora (cuchilla de afeitar)", "Pensar en todo lo que voy a hacer, lo hago en mi cuarto y escucho música".

En lo concerniente a las conductas durante la autolesión, 5 reportan el procedimiento (tomar pastillas o veneno):"Ese día discutí con mi padrastro, y él me gritó (pues mátese), luego se fue con mi hermana; y mezclé el veneno con mazamorra y lo ingeri", "Tomaba agua y tomaba las pastillas", "Estaba sentado en el patio de la casa, esperando que me hiciera efecto"; 2 se golpean por ira: "Le pego a las paredes", "No me oculto lo hago en el salón enfrente de los demás, algunos saben y no dicen nada y eso me molesta"; 10 sólo se cortan: "Me quedaba quieta en mi cama, lo hice mientras mi mamá dormía, lo hice 5 veces", "Sólo cortarse y sentir el dolor físico"; y 4 lloran: "Cantar y llorar por lo que hacía”, "Nada, llorar algunas veces porque duele".

Otro reporte es el de las conductas después de la autolesión; 4 escuchan música: "Me senté en el sofá a escuchar música", "Lloro escuchando música y me duermo"; 3 chatean: "Cojo el celular y me pongo a chatear", "Por el whatsap le cuento a mi mejor amiga"; 2 regresan a la clase: "Enfrentar el llamado del profe porque un compañero me vio y le contó y luego enfrentar a mi mamá"., "Del baño me regreso al salón de clase"; 2 reportan no hacer nada: "Sigo normal como si nada hubiera pasado", "Como lo hago sola en la casa, sigo haciendo lo de todos los

días"; 2 se van a comer: "A comer sola", "Busco qué comer"; 7 se van a taparse las cortadas: "Me ponía un saco para tapar las muñecas y jean para las piernas", "Trataba de ocultar mis heridas, que mis abuelos no se dieran cuenta porque me daba miedo"; uno vomita: "Escucho lo que hablan mis amigos y voy a vomitar, siempre voy 
a vomitar luego de cada borrachera"; y otro duerme: "Antes de dormirme me pongo brava y me desquito con las almohadas y escondo las cuchillas".

En ésta dimensión surgieron reportes adicionales: "Hubo un intento suicida con ingesta de veneno, producto de este evento quedó en coma y sufrió parálisis del cuerpo, actualmente se encuentra recuperándose"; "Informa que practica el cutting, lo ha hecho 5 veces, con bisturí o cuchilla de afeitar y simultáneamente y un amigo me dijo que era más facíl hacerlo si consumía dick, marihuana, cripy y popper así como gotas formuladas por el psiquiatra".

Se destaca que en las instituciones educativas de la ciudad de Ibagué existe el fenómeno del parasuicidio (acto de autoagresión sin deseo de morir) porque los adolescentes están atrapados en sus lógicas etareas replicando patrones generados por las historias familiares, modelos de sus pares, desesperanza por su condición social y rasgos de personalidad, aclarando que no hubo ningún caso de la población LGBTI ni con diagnóstico de trastorno mental, resultados que van en la misma dirección del informe de la OPS/OMS (2014) y del estudio de GallegosSantos, Casapia, \& Rivera, (2018)., en el cual encontraron datos suficientes para considerar que los niños parasuicidas poseen una historia de problemas socio personales, básicamente familiares, que los induce a intentar suicidarse dentro del campo de conceptualización de la muerte que su edad les proporciona; éstos supuestos son reforzados por la OPS cuando reportan que, en los adolescentes, un mayor riesgo depende del bajo nivel económico y educativo, así como del desempleo en la familia, los que pueden considerarse factores de riesgo.

La conducta de autolesión, lejos de ser un enemigo invisible, se muestra a los ojos como una realidad, como un síntoma de un problema de salud pública en el país y concretamente en la ciudad de lbagué; esto en coherencia con Gallego-Santos et al., (2018) quienes citan a Purington (2010), planteando que la autolesión está aumentando en prevalencia, y el contagio contribuye a la difusión de la autolesión en adolescentes, ellos suelen "probar" diferentes roles, uno tras otro, buscando los comportamientos aceptables, los mecanismos de supervivencia y sistemas de apoyo. Cuando un compañero encuentra consuelo en la autolesión, la información podrá ser compartida con otro compañero del grupo, quien lo puede animar a intentarlo.

Los estudiantes que experimentan aislamiento en su vida son más vulnerables al suicidio que los que tienen vínculos sociales fuertes con otras personas con mayor frecuencia en las mujeres, datos que coinciden con De la Fuente (2018), quien en su estudio destaca que ser mujer ha sido identificado como un factor de riesgo para presentar autolesiones; en ellas tienen gran impacto la negligencia emocional por parte de sus progenitores, una relación inestable con el padre y experimentar abuso sexual en la infancia, captar la atención de alguien, la soledad o muerte de un ser querido.

Esta investigación aporta a la definición de parasuicidio o conducta autolesiva, aquellos procedimientos que no llevan intención de muerte sino la búsqueda intencional para compensar y/o aliviar los sentimientos de desesperanza, ira, soledad o tristeza, que al hacerlo repetitivamente, lo convierte en un indicador de alto riesgo para finalmente consumar la muerte. Flores et al (2018), presentan una definición similar para las conductas autolesivas "consisten en realizarse daño físico sin intención suicida" y la entienden como un proceso de adaptación. Con el mismo enfoque Villarroel (2013), enuncia que las conductas autolesivas son estrategias de afrontamiento del stress emocional como medio útil para regular afectos negativos intolerables producto de una inestabilidad emocional.

Finalmente se pudo conocer, con ésta investigación, los pensamientos, emociones y acciones de la población adolescente con prácticas de autolesión e intento suicida, aportando nuevos saberes y haciendo visible las realidades de los actores de dicho fenómeno, con la intención de no desconocerlos en futuras propuestas de investigación, intervención y formulaciones para políticas públicas, como el llamado que hacen los 
investigadores colombianos Caycedo et al. (2009) y Toro et al. (2018) para fortalecer los factores protectores y vigilar los factores de riesgo que pueden ayudar a prevenir y/o detectar tempranamente la población en riesgo de suicidio tales como: efecto de imitación o efecto Werther "fenómeno mediante el cual la observación o notificación del suicidio de una persona conduce a otra a intentar imitar dicha muerte" (Ramírez, 2015), la pérdida de una persona significante, antecedentes de depresión en la familia, muerte o separación de los padres, desempleo, bajo rendimiento escolar, rompimiento de una relación sentimental, dificultades afectivas, baja autoestima, pocas habilidades sociales o rechazo social.

Urge entonces, la necesidad de implementar acciones interinstitucionales en el abordaje de esta problemática social, orientadas a la prevención e intervención oportuna y eficaz del parasuicidio como factor precipitante del suicidio. Las autolesiones son un problema que requieren atención urgente en todos los sectores: educativo, salud, protección, justicia social, oportunidad laboral para las familias, recreación organizada que debe ser contemplado en las políticas públicas relacionadas con infancia y adolescencia, con el objeto de definir lineamientos para su abordaje como problema social como lo plantean Toro (2018) y Scilletta (2009).

Cualquier política pública debe hacer énfasis en la intervención reforzando los factores protectores para que no se presente el fenómeno de autolesionarse por parte de los adolescentes como lo reportan Medina-Pérez, Londoño Osorio y Orozco Roa (2008) y la Secretaria de Salud, (2011), donde la intervención familiar desde la provisión de los recursos básicos para la subsistencia, el cuidado del hogar, la protección de los jóvenes, la orientación y promoción del desarrollo físico y psicológico de estos, y la defensa y apoyo de la causa juvenil ante la comunidad o la sociedad mayor. La familia y la escuela proporcionan elementos de riesgo o de protección, según el caso, al encontrarse que la principal causa para idear el suicidio tiene como base la familia, pues se considera que esta se halla en decadencia y crisis (Villarroel et al., 2013). En la misma dirección Piedrahita et al. (2012) resaltan que la intervención educativa, posibilitó la identificación de los factores de riesgo en los adolescentes y mostró una significativa efectividad al aumentar el nivel de conocimientos en los padres y educadores.

\section{Conclusiones}

Entender esta pluralidad de factores comprometidos en el fenómeno, implica imaginar estrategias autolesivas presentes en los púberes y adolescentes en condiciones vulnerables que están casi al límite con la pobreza y corriendo riesgos con su integridad física y psicológica; reforzada por el modelo entre pares. Esta realidad local se explica desde una perspectiva multifactorial al considerarlo como una patología psicosocial, consecuencia y expresión de los conflictos que sufre ésta etapa generacional donde es importante resaltar que el rol familiar está doblemente presente: como factor protector y como factor desencadenante que plasman con mucho énfasis los reportes de éstos púberes y adolescentes que han experimentado conductas parasuicidas, las acciones de cómo estos actores perciben antes y después del hecho, la conducta autolesiva.

La intención fue traer estas vivencias y comprender las realidades en su multicausalidad, en su contexto y hacer un acercamiento a una población muy joven, para comprender su realidad y la forma en cómo la interpretan, la viven y la reconstruyen en su vida cotidiana. Por este motivo, se hizo indispensable que fueran ellos los que expresaran por medio de cuestionarios y la entrevista semiestructurada, cuales son las variables que rodean esas prácticas, sus posibles causas y los impactos que tiene en sus vidas y las de sus familias, detectando la presencia de la desesperanza, la ira y la tristeza como emociones transversales al antes, durante y después de las conductas autodestructivas.

En este sentido se hace necesario implementar acciones psicosociales para prevenir las conductas autolesivas o parasuicidio como tener en cuenta cualquier cambio repentino o dramático que afecte el desempeño de un adolescente, entre ellos, falta de interés en las actividades cotidianas, alteración del rendimiento académico, 
ausencias inexplicadas, cambio en las conductas alimentarias, descuido en la presentación personal, aburrimiento constante, dificultad de concentración, alteración en el patrón de sueño, manifestaciones de emociones contenidas, preocupación acerca de la muerte, dibujos o notas sobre la muerte, puesto que cualquier cambio o manifestación a nivel cognitivo, comportamental o emocional son una alerta para la detección temprana.

\section{Referencias bibliográficas}

Acevedo, Y., Lizcano, J. y Serrano, H. (2020). Las conductas autolesivas en los adolescentes una revisión documental. Trabajo de Grado. Universidad Cooperativa de Colombia. Recuperado de: https://repository.ucc.edu.co/bitstream/20.500.12494/18046/1/2020_conductas_autolesivas.pdf

Alarcón-Velásquez, Y., Bahamón, J., Trejos, A., González-Gutiérrez, O., Rubio-Castro, R., Hernández-Cervantes, Q., y Gómez-Maqueo, E. (2019). Propiedades Psicométricas del Inventario de Riesgo Suicida (IRISA). Revista Iberoamericana de Diagnóstico y Evaluación - e Avaliação Psicológica, 51 (2), 87-98. Recuperado de: https://www.aidep.org/sites/default/files/2019-04/RIDEP51-Art7.pdf

American Psychiatric Association (2014). Diagnostic and Statistical Manual of Mental Disorders (DSM)-5 (2014). Quinta Edición. EAN: 9788498358100. Recuperado de:

http://www.medicapanamericana.com/Libros/Libro/4949/DSM5-Manual-Diagnostico-y-Estadistico-de-losTrastornos-Mentales.html?gclid=CID90P6an9QCFcdDhgod2loFlg

Beaven, N., Ciapara, Campa, Álvarez, R. y Valenzuela, B. (2018). Inclusión educativa: Factores psicosociales asociados a conducta suicida en adolescentes. Revista de Ciencias Sociales e Investigación Social, 23, 185207. Recuperado de: http://revistaprismasocial.es/article/view/2770/2916

Caycedo, A., Arenas, M., Benítez, M. Cavanzo, P., Leal, G. y Guzmán, Y. (2009). Características psicosociales y familiares relacionadas con intento de suicidio en una población adolescente en Bogotá - 2009.

Recuperado de:

http://personaybioetica.unisabana.edu.co/index.php/personaybioetica/article/viewArticle/1807/2424

Colón, T., Escobar, A., Santacoloma, A., Granados-García, A., Moreno, S. y Silva, L. (2019). Caracterización psicosocial y de motivos de consulta de la población asistente a 28 Centros de Atención Psicológica Universitarios en Colombia. Universitas Psychologica, 18 (4). Recuperado de: https://revistas.javeriana.edu.co/files-articulos/UPSY/18-4\%20(2019)/64761787020/

De la Fuente, M. (2018). Autolesiones en la adolescencia. Universidad de Valladolid, España. UVaDOC. Recuperado de: http://uvadoc.uva.es/handle/10324/30252

Departamento Administrativo Nacional de Estadística (2017). Boletín de salud mental: conducta suicida. Recuperado de: https://www.minsalud.gov.co/sites/rid/Lists/BibliotecaDigital/RIDE/VS/PP/ENT/boletinconducta-suicida.pdf

Domínguez, J. (2015). Suicidios en Colombia, cifras 2013. Recuperado de: https://www.sura.com/blogs/calidadde-vida/suicidios-colombia.aspx

Flores, M., Cancino, M. y Figueroa, M. (2018). Revisión sistemática sobre conductas autolesivas sin intención suicida en adolescentes. Revista Cubana de Salud Pública, 44 (4), 200-216. Recuperado de: http://www.redalyc.org/jatsRepo/214/21458870016/21458870016.pdf 
Gallegos-Santos, M., Casapia, Y., \& Rivera, R. (2018). Estilos de personalidad y autolesiones en adolescentes de la ciudad de Arequipa. Interacciones, 4(2), 143-151. Recuperado de:

https://ojs.revistainteracciones.com/index.php/ojs/article/view/106/html

García-Nieto, R., Parra Uribe, I., Palao, D., Lopez-Castroman, J. et al., .... y Baca-García, E. (2003), Protocolo breve de evaluación del suicidio: fiabilidad interexaminadores. Revista de Psiquiatría y Salud Mental, 5 (1), 24-36. doi: 10.1016/j.rpsm.2011.10.001

Gaviria, C. y Torres, A. (2018). Prevención de la conducta suicida en adolescentes entre los 12-18 años de edad de la ciudad de Bogotá. Trabajo de Grado, Universidad Cooperativa de Colombia.

González, L.., Vasco- Hurtado, I., Nieto- Betancurt, L. (2016). Revisión de la literatura sobre el papel del afrontamiento en las autolesiones no suicidas en adolescentes. Cuadernos Hispanoamericanos de Psicología, 16, (1), 41-56. Recuperado de: https://dialnet.unirioja.es/servlet/articulo?codigo=5855292

Instituto Nacional de Medicina Legal y Forenses (2015). Informe Forensis de Medicina Legal: Estadística sobre violencia en Colombia. Recuperado de: http://www.medicinalegal.gov.co/el-instituto/-

/asset_publisher/4Of1Zx8ChtVP/content/forensis-2015-informacion-estadistica-sobre-violencia-encolombia;jsessionid=723AEA36DA4A937937DBF4529C372208

Instituto Nacional de Medicina Legal y Forenses (2019). Forensis. Recuperado de:

https://www.medicinalegal.gov.co/cifras-estadisticas/forensis

Londoño Muriel, V. y Cañón Buitrago, C. (2020). Factores de riesgo para conducta suicida en adolescentes escolarizados: revisión de tema. Archivos de Medicina (Col), 20 (2). doi:

https://doi.org/10.30554/archmed.20.2.3582

Medina-Pérez, O., Londoño Osorio, A. y Orozco Roa, C. (2008). Caracterización del suicidio en adolescentes del Quindío, Colombia (1989-2008). Universitas Médica, 53 (3), 249-259. Recuperado de: https://www.redalyc.org/pdf/2310/231026412003.pdf

Ministerio de Salud y Protección Social (2012). Plan Decenal de Salud Pública. Recuperado de: https://www.minsalud.gov.co/sites/rid/Lists/BibliotecaDigital/RIDE/VS/ED/PSP/IMP_4feb+ABCminsalud.p df

Monge, J. (2013). Salud del adolescente. OPS/OMS, Washington, D.C. Boletín Clínico de la Secretaría de Salud Pública del Estado de Sonora, México, 15(2), 77-85.

Organización Mundial de la Salud (2006). Prevención del suicidio. Un instrumento en el trabajo. Departamento de Salud Mental y Abuso de Sustancias Manejo de Trastornos Mentales y Cerebrales. Ginebra. Recuperado de: http://www.who.int/mental_health/prevention/suicide/resource_work_spanish.pdf

Organización Mundial de la Salud (2018). Suicidio: Datos y cifras. 24 de agosto de 2018. Recuperado de: https://www.who.int/es/news-room/fact-sheets/detail/suicide

Organización Mundial de la Salud (2019). Suicidio. Recuperado de: https://www.who.int/news-room/factsheets/detail/suicide

Organización Mundial de la Salud (2014). Programa de Acción para Superar las Brechas en Salud Mental, mhGAP. Recuperado de: https://www.who.int/mental_health/publications/9789242596205/es/ 
Organización Panamericana de la Salud - Organización Mundial de la Salud (2014). Mortalidad por suicidio en las Américas. Informe Regional. Recuperado de: https://www.paho.org/es/documentos/mortalidad-porsuicidio-americas-informe-regional-2014

Piedrahita, L., Paz, K. y Romero. A. (2012). Estrategia de intervención para la prevención del suicidio en adolescentes: La escuela como contexto. Hacia la Promoción de la Salud, 17 (2), 136 - 148. Recuperado de: https://www.redalyc.org/pdf/3091/309126826010.pdf

Plan de Desarrollo Municipal de la ciudad de Ibagué (2016 -2019) (2016). No segregar, no depredar no robar. Recuperado de: http://www.ibague.gov.co/portal/admin/archivos/publicaciones/2016/14981-PLA20161104.pdf

Ramírez, R. (2015). El tratamiento del suicidio en la prensa española: ¿efecto werther o efecto papageno?. Rev. Asoc. Esp. Neuropsiq., 2015; 35 (125), 123-134. doi: 10.4321/S0211-57352015000100009. Recuperado de: http://scielo.isciii.es/pdf/neuropsiq/v35n125/original08.pdf

Red de Salud de Cuba (2018). Los jóvenes y la salud mental en un mundo en transformación. Recuperado de http://www.sld.cu/anuncio/2018/10/10/los-jovenes-y-la-salud-mental-en-un-mundo-en-transformacion

Rodríguez Molinero, L. (2017). El adolescente y su entorno. Pediatría Integral, 21 (4), 261-269. Recuperado de: https://www.pediatriaintegral.es/publicacion-2017-06/el-adolescente-y-su-entorno-familia-amigosescuela-y-medios/

Sanderson, M. (2020). Predicting death by suicide following an emergency. Xx, 8 (3), e672. doi: http://dx.doi.org/10.20511/pyr2020.v8n3.672.

Sandoval, C. (2010). Investigación cualitativa. Programa de especialización en Teoría, métodos y técnicas en investigación social. Instituto Colombiano para el Fomento de la Educación Superior ICFES. ARFO Editores e Impresores Ltda. Bogotá, Colombia.

Scilletta, D. (2009). Autolesiones mediante cortes reiterados en piel. Subjetividad y procesos cognitivos. Universidad de Ciencias Empresariales y Sociales (UCES), 13(2), 183-197. Recuperado de: http://dspace.uces.edu.ar:8180/xmlui/handle/123456789/938

Secretaría Distrital de Salud. (2011). Guía operativa, plan de intervenciones colectivas. Bogotá, Colombia.

Soto Salcedo, A., Villaroel Grüner, P., \& Véliz Burgos, A. (2020). Factores que intervienen en riesgo suicida y parasuicida en jóvenes Chilenos. Propósitos y Representaciones, 8(3), e672.

https://dx.doi.org/10.20511/pyr2020.v8n3.672

Suárez Colorado, Yuly, \& Campo-Arias, Adalberto. (2019). Asociación entre apego y riesgo suicida en adolescentes escolarizados de Colombia. Revista chilena de pediatría, 90(4), 392-398. https://dx.doi.org/10.32641/rchped.v90i4.985

Tomko, R., Prisciandaro, J., Falls, S., \& Magid, V (2016). The Structure of the UPPS-R-Child Impulsivity Scale and its relations with substance use outcomes among treatment-seeking adolescents. Published online in Drug Alcohol Dependence, 1. Recuperado de: https://www.ncbi.nlm.nih.gov/pmc/articles/PMC4799694/

Toro, R., Vianchá, M., Martínez, L. y Pérez, M. (2018). Conducta suicida. Factores psicosociales y prevención. Editorial Universidad de Boyacá. Edición No. 1. Recuperado de: https://www.uniboyaca.edu.co/es/centroinformacion/publicaciones/conducta-suicida-en-boyaca-factores-psicosociales-y-prevencion 
Vasilachis, I. (2006). Estrategias de investigación cualitativa. Gedisa Editorial Barcelona, España. Recuperado de: http://jbposgrado.org/icuali/investigacion\%20cualitativa.pdf

Villarroel, J., Jerez, S., Montenegro, A., Montes A., Igor, M., \& Silva, H. (2013). Conductas autolesivas no suicidas en la práctica clínica: Primera parte: conceptualización y diagnóstico. Revista Chilena de Neuropsiquiatría, 51(1), 38-45. doi: https://dx.doi.org/10.4067/S0717-92272013000100006

\section{Anexos}

\section{Anexo 1}

TEST IRSA

\section{Estimado(a) estudiante}

El test que a continuación vas a llenar, pretende conocer algunos aspectos de tu vida, de lo que sientes o piensas.

Contesta todas las preguntas que se te presentan a continuación, marcando con una ( $\mathrm{x}$ ) el cuadrito contiguo a la respuesta (Sí o No) que consideras se aplica mejor a tu situación. No debes escribir tu nombre. Tus respuestas serán confidenciales y solo serán vistas por los profesionales de salud que realizan el estudio. No permitas que nadie más observe o te sugieran las respuestas. Agradecemos tu confianza e interés.

NO EXISTEN RESPUESTAS BUENAS, MALAS, CORRECTAS O INCORRECTAS. TODAS SON IMPORTANTES Edad Fecha Sexo ( ) Masculino ( ) Femenino

¿Dóndevives?

\section{ITEM}

1. En el último año mis padres se separaron o divorciaron

2. En los últimos seis meses he consumido drogas prohibidas por la ley (marihuana, cocaína, crack u otras)

3. La mayoría de la gente me aprecia

4. Considero que las cosas estarían mejor si yo no estuviera

5. Tiendo a ser muy impulsivo la mayor parte del tiempo

6. Casi siempre me siento enojado

7. Soy una persona valiosa

8. He pensado en un plan o método para matarme

9. A menudo me siento muy presionado y con demasiadas responsabilidades

10. Algún amigo o familiar cercano murió recientemente

11. Siento que la vida vale la pena

12. Pienso en el suicidio como una posible solución a los problemas

13. En algún momento de mi vida, he intentado hacerme daño

14. En los últimos seis meses he consumido bebidas alcohólicas en exceso

15. Hay gente que se interesa por mí y por lo que me pasa

16. A menudo me siento sólo y aislado de los demás

17. Me siento incapaz de hacer bien las cosas y salir adelante

18. Tengo amigos con quienes contar

19. Me siento mal por no poder llenar las expectativas de los demás 
20. He pensado en el modo de hacerme daño

21. Siento que puedo confiar en las personas de mi familia

22. Recientemente perdí a alguien que quería mucho

23. A veces pienso que yo merecería morir

24. He sufrido abuso sexual

25. En mi familia con frecuencia hay conflictos y peleas

26. Tiendo a reaccionar violentamente cuando algo no sale como quiero

27. A menudo siento que no podré ser feliz

28. Tengo planes para el futuro

29. Me siento culpable la mayor parte del tiempo

30. Pienso que soy un perdedor

31. Me extrañarían si yo falto

32. He buscado métodos para matarme en revistas, Internet $u$ otros medios

33. Con frecuencia me siento muy confundido

34. Estoy muy dolido porque mi relación de pareja terminó en fracaso

35. La mayor parte del tiempo me siento bien

36. Pienso a menudo que las cosas están mal y seguirán siempre así

37. En mi familia hemos sufrido violencia doméstica

38. En mi casa a menudo me castigan golpeándome

39. Pienso que soy capaz de hacer casi todo lo que me proponga

40. La mayoría del tiempo siento que soy una persona valiosa

41. Me es difícil concentrarme por mis preocupaciones

42. Me he expuesto a situaciones que me ponen al borde de la muerte

43. Me agreden verbal o físicamente en el lugar donde estudio o trabajo

44. Pienso que he hecho algo que no tiene perdón

45. Algún miembro de mi familia ha intentado matarse o se ha suicidado

46. Yo cuento con el apoyo de mi familia

47. Últimamente he estado pensando en hacerme daño

48. Tengo problemas para dormir

49. La mayor parte del tiempo me siento triste

50. La mayoría de las personas están mejor que yo

51. Tengo acceso a armas de fuego

52. Cuando me enojo, me corto o hago cosas que me dañan

53. He pensado alguna vez que lo mejor sería estar muerto

54. Mis compañeros de estudio o trabajo me hacen la vida imposible

55. Últimamente he tenido muchos problemas en mi familia

56. He pensado últimamente que la vida no merece la pena ser vivida

57. Tengo muchas razones para vivir

58. Un amigo o amiga se ha suicidado 
59. Constantemente fracaso en mis estudios

60. Si me siento mal busco a alguien que me escuche y me ayude

61. Estoy más irritable que de costumbre

62. Formo parte de algún grupo u organización de jóvenes

63. A menudo no siento deseos de comer.

64. Siento que mi situación es desesperante

65. Hablo frecuentemente de la muerte y/o de los deseos de estar muerto

66. Me he sentido humillado y solo en el lugar donde estudio o trabajo

67. Aprendo mucho de mis propios problemas

68. Los demás descansarían con mi muerte

69. He pensado en matarme

70. Me han expulsado del colegio

71. Soy vengativo y rencoroso

72. Me preocupo demasiado por las cosas

73. Hago amigos con facilidad

74. He tenido problemas por mi preferencia u orientación sexual

75. He escrito cartas de despedida por si decido acabar con mi vida

76. Soy tan bueno en lo que hago como mis compañeros o amigos

77. Recientemente terminó mi relación de pareja

78. Soy capaz de encontrar una solución a mis problemas

79. Sé que puedo tener una vida feliz

80. He intentado suicidarme

81. A menudo participo en peleas a golpes

82. Tiendo a hacer cosas que me ponen en peligro

83. Últimamente estoy cansado y sin energías

84. Con frecuencia pienso antes de actuar

85. Descanso poco cuando duermo.

86. A menudo disfruto con lo que hago

87. A menudo tengo deseos de morirme

88. Actualmente estoy muy molesto con alguien especial para mi

89. Me afecta mucho no ser como quisiera

90. Me han tenido que atender u hospitalizar por problemas psicológicos

91. Sé quién puede ayudarme en momentos de crisis

92. He tenido conflictos con la policía

93. Tengo amigos en quien apoyarme

94. Últimamente logro concentrarme en lo que hago

95. Me siento inútil.

96. Tengo la confianza de contarles mis problemas a mis familiares

97. Siento que no le caigo bien a la gente 
98. Siento que pierdo el control cuando me enojo

99. He sufrido una experiencia traumática recientemente

100. Sufro de alguna enfermedad que me angustia o me desespera constantemente

101. Cuento con un grupo de amigos para divertirme

102. Alguna vez he pensado en hacerme daño, luego de terminar una relación de pareja

103. Siento que vine al mundo a sufrir

104. Me molesta que otros se interesen por mí

105. Me gusta sentirme mal

106. Siento que no soy tan bueno como otros

107. Cuando estoy enojado o frustrado busco alguna actividad recreativa para desahogarme

\section{Anexo 2}

Escala de ideación suicida de Beck

\section{CARACTERÍSTICAS DE LA ACTITUD HACIA LA VIDA / MUERTE.}

1.- Deseo de vivir.

! Moderado a intenso ! Débil ! Ninguno

2.- Deseo de morir.

! Moderado a intenso ! Débil ! Ninguno

3.- Razones para vivir / morir.

! Las razones para vivir son superiores a las de morir

! Iguales

! Las razones para morir son superiores a las de vivir

4.- Deseo de realizar un intento activo de suicidio.

! Moderado a intenso ! Débil ! Ninguno

5.- Intento pasivo de suicidio.

! Tomaría precauciones para salvar su vida

! Dejaría su vida / muerte en manos del azar (por ej.: cruzar descuidadamente una calle muy transitada)

! Evitaría los pasos necesarios para salvar o mantener su vida (por ej.: un diabético que deja de tomar la insulina)

\section{II.- CARACTERÍSTICAS DE LOS PENSAMIENTOS / DESEOS SUICIDAS.}

6.- Dimensión temporal: duración.

! Breve, períodos pasajeros

! Períodos más largos

! Continuo (crónico), o casi continuo

7.- Dimensión temporal: frecuencia.

! Rara, ocasional! Intermitente ! Persistente o continuo

8.- Actitud hacia el pensamiento / deseo.

! Rechazo! Ambivalencia, indiferencia ! Aceptación 
9.- Control sobre la acción del suicidio o el deseo de llevarlo a cabo.

! Tiene sensación de control

! No tiene seguridad de control

! No tiene sensación de control

10.- Factores disuasorios del intento activo. El terapeuta debe anotar cuáles son (ej.: familia, religión, posibilidad de quedar seriamente lesionado si fracasa el intento, irreversibilidad).

! No intentaría el suicidio debido a algún factor disuasorio

! Los factores disuasorios tienen cierta influencia

! Influencia mínima o nula de los factores disuasorios

Si existen factores disuasorios, indicarlos:

11.- Razones del proyecto de intento.

! Manipular el medio, atraer atención, venganza

! Combinación de ambos

! Escapar, acabar, resolver problemas

III.- CARACTERÍSTICAS DEL PROYECTO DE INTENTO.

12.- Método: especificación / planes.

! No los ha considerado

! Los ha considerado, pero sin detalles específicos

! Los detalles están especificados / bien formulados

13.- Método: accesibilidad / oportunidad.

! Método no disponible; no hay oportunidad

! El método llevaría tiempo / esfuerzo; la oportunidad no es accesible fácilmente.

! Método y oportunidad accesible

! Oportunidades o accesibilidad futura del método proyectado

14.- Sensación de "capacidad" para llevar a cabo el intento.

! No tiene coraje, demasiado débil, temeroso, incompetente

! Inseguro de su coraje, competencia

! Seguro de su competencia, coraje

15.- Expectativas / anticipación de un intento real.

! No

! Incierto, no seguro

! Sí

\section{IV.- REALIZACIÓN DEL INTENTO PROYECTADO.}

16.- Preparación real.

! Ninguna

! Parcial (ej.: empezar a recoger píldoras)

! Completa (ej.: tener las píldoras, la navaja, la pistola cargada)

17.- Notas acerca del suicidio.

! No escribió ninguna nota

! Empezada pero no completada; solamente pensó en dejarla 


\section{! Completada}

18.- Preparativos finales ante la anticipación de la muerte (ej.: seguro,

testamento, donaciones).

! Ninguno

! Pensamientos de dejar algunos asuntos arreglados

! Hacer planes definitivos o dejarlo todo arreglado

19.- Engaño / encubrimiento del intento proyectado (se refiere a la comunicación de su idea al terapeuta).

! Reveló estas ideas abiertamente

! Fue reacio a revelarlas

! Intentó engañar, encubrir, mentir

\section{FACTORES DE FONDO.}

20.- Intentos previos de suicidio.

! Ninguno

! Uno

! Más de uno

21.- Intención de morir asociada al último intento.

! Baja

! Moderada; ambivalente, insegura

! Alta

\section{PUNTUACIÓN TOTAL:}

Actitud ante la vida/muerte:

Pensamientos/deseos suicidas:

Proyecto de intento:

Intento proyectado:

Factores disuasorios:

Intentos previos:

\section{Anexo 3}

Entrevista sociodemográfico y áreas de ajuste cognitiva, emocional y conductual

\section{ENTREVISTA SEMI ESTRUCTURADA}

"Caracterización de la experiencia del parasuicidio en púberes y adolescentes de instituciones educativas públicas y privadas de la ciudad de Ibagué"

\section{ETAPAS}

PRESENTACIÓN:

- Breve reseña de Quién es el entrevistador (Quién es y de dónde viene)

- Se invita al entrevistado a hacer su presentación

\section{ENCUADRE:}

- Se presenta la actividad que se llevará a cabo 
- Se articula con la fase de aplicación de pruebas

RAPPORT

- Se harán preguntas exploratorias de la cotidianidad del adolescente

- (¿Cómo se encuentra el día de hoy?, como le está yendo en el colegio?, ¿el clima? Éstas de acuerdo a la comodidad y el estilo del entrevistador)

- Aplicación del Cuestionario C Riesgo Suicida

- DESARROLLO DE LA ENTREVISTA

- Dimensión Cognitiva

1. ¿Qué información conoces frente a las conductas auto lesivas?

2. ¿Crees que tus seres queridos estarían mejor sin ti?

3. ¿Qué pensamientos tienes antes, durante y después de la conducta auto lesiva?

4. ¿Has pensado nuevamente en causarte daño físico? Por qué?

- Dimensión emocional

5. ¿Cuáles son los sentimientos o emociones que te invaden antes, durante y después de la conducta auto lesiva?

6. ¿Crees que tus seres queridos estarían mejor sin ti? ¿Por qué?

7. ¿Estos sentimientos de autolesión están relacionados con: 1. Familia. 2. Colegio. 3. Amigos. 4. Barrio. 5. Otro?

8. ¿Te sientes importante y amado por alguien?

- Dimensión comportamental

9. ¿Puedes describir los comportamientos antes, durante y después de la conducta auto lesiva?

10. ¿Has pensando nuevamente en causarte daño físico y por qué?

11. ¿Buscas información frente a las conductas auto lesiva? (Es decir medios de comunicación, redes sociales, amigos, y otros).

12. ¿¿suele aislarse durante los días u horas previas a la conducta autolesiva?

Esta obra está bajo una Licencia Creative Commons Attribución-NoCommercial 4.0 International

\section{$(\mathrm{cc})$ EY-NO}

